\title{
EVOLUTION TOWARDS THE WHITE-DWARF STAGE
}

\author{
S. C. VILA \\ (Indiana University, U.S.A.)
}

We have followed the evolution of stellar models of 1 and $1.2 M_{\odot}$ from a nondegenerate configuration to the stages where degeneracy becomes important and eventually causes the star to become a white dwarf. By detailed computer calculation using an evolution program kindly lent to us by Schwarzschild and Härm, we have obtained evolution sequences of stars of the mentioned masses with a core composition of $80 \% \mathrm{O}, 10 \% \mathrm{Ne}$ and $10 \% \mathrm{Mg}$ and a thin outer envelope of $\mathrm{He}\left(10^{-6}\right.$ of total mass) under the assumption of energy loss due to photons alone, and also under the assumption of losses due to photons plus neutrinos.

These models were intended to represent the remnant cores of more massive stars that have lost their outer layers. Further evolution reduces to contraction and release of the gravitational and thermal energies but some interesting features appear where the neutrino losses are included that we think might be related to the evolution of the stellar nuclei of planetary nebulae.

In the calculation of the models matter has been assumed to be completely ionized, its pressure and entropy being the sum of the ion, electron and radiation components. The opacities were kindly calculated by A.N. Cox using the Los Alamos opacity program and were extended to the electron conduction region using the work of Mestel (1950).

The neutrino reactions included were: pair annihilation of electron pairs into neutrinos, photoneutrinos and plasma neutrinos. All these stem from the hypothesis of universality of weak interactions for which there is no direct experimental confirmation, but in whose existence there is a confident belief among physicists.

Evolution with no neutrinos proceeds for a time almost as a homologous contraction with constant luminosity. When degeneracy becomes important the electron pressure stops the contraction and electron conduction causes the core to become isothermal. Further evolution asymptotically approaches a line of cooling at constant radius. The evolution with neutrino losses included (started from the same stellar model) proceeds in a similar way until the central temperature is high enough for an appreciable neutrino production. In that case, neutrinos are produced at the center and their escape and subsequent energy loss causes the core to evolve much more rapidly towards the degenerate state. The central temperature falls faster than the envelope temperature because as the equation of state of a degenerate gas is almost insensitive to the temperature, the depletion of energy by neutrino emission is not compensated

Osterbrock and O'Dell (eds.), Planetary Nebulae, 421-422. (O I.A.U. 
by contraction and subsequent release of gravitational energy. This causes energy to flow from the envelope to the core to supply the neutrino losses. This sudden cooling and contraction of the core causes the outer layers to collapse and release gravitational energy at a fast rate. This causes an increased luminosity and effective temperature as reflected in the HR diagram.

The evolution line obtained for the neutrino-star models invites comparison with the evolution of the central stars of planetary nebulae recently proposed by Harman and Seaton (1964) and Seaton (1966). Though the luminosities are of the same order of magnitude, the effective temperatures in the calculated models are systematically higher. Presumably a more realistic model with an extended envelope of $\mathrm{H}$ or $\mathrm{He}$ could reduce the temperatures while keeping the same luminosities.

The most suggestive feature in this comparison is that the lifetime estimated by Seaton (1966) for the nuclei of planetary nebulae is on the order of $10^{4}$ years while the lifetime obtained by gravitational collapse alone is hundreds of times greater.

On the negative side, we must point out that in our scheme there is no explanation for the ejection of the planetary shell, but perhaps such a phenomenon can result from a not too deep shell of $\mathrm{H}$ or $\mathrm{He}$ that is ignited by the rise of envelope temperature in the neutrino-collapse phase. This is at present being investigated.

Although we have presented no conclusive proof, we would like to suggest that the late stages of evolution of solar-mass stars with neutrino losses might provide an explanation for the evolution, of central stars of planetary nebulae and at the same time might provide evidence in favor of the universality of weak interactions between Fermi particles.

Further details of the models, as well as a figure showing tracks in the HR diagrams are given in Vila (1966).

\section{References}

Harman, R.J., Seaton, M.J. (1964) Astrophys. J., 140, 824.

Mestel, L. (1950) Proc. Camb. Phil. Soc., 46, 331.

Seaton, M.J. (1966) Mon. Not. R. Astr. Soc., 132, 113.

Vila, S.C. (1966) Astrophys. J., 146, 437. 